\title{
Generalized Proportional Fair (GPF) Scheduler for LTE-A
}

\author{
Samuel O. Aramide, Basel Barakat, Yi Wang, Simeon \\ Keates. \\ Department of Engineering Science, \\ Faculty of Engineering and Science, \\ University of Greenwich, United Kingdom \\ Email: \{ ao2659e ,bb141, yi.wang, \\ s.keates\}@greenwich.ac.uk
}

\author{
Kamran Arshad \\ Department of Electrical Engineering, \\ Ajman University, UAE \\ Email: k.arshad@ajman.ac.ae
}

\begin{abstract}
The growth of wireless traffic and the demand for higher data rates motivated researchers around the world to enhance the Long Term Evolution-Advanced (LTE-A) performance. Recently, a considerable amount of the research had been done to optimise the packet schedulers. The packet schedulers distribute the radio resources among users to increase spectrum efficiency and network performance. In this paper, a Generalized Proportional Fair (GPF) scheduler is used to enhance the scheduler performance compared to the other conventional schedulers. The GPF scheduler performance is compared in terms of users' throughput, energy efficiency, spectral efficiency and fairness using system level simulations. The simulation results show that the proposed scheduler outperforms the conventional schedulers proposed for LTE-A.
\end{abstract}

\section{INTRODUCTION}

The Radio Resource Management (RRM) techniques, such as packet scheduler, plays a fundamental role in the enhancement of the users Quality of Service (QoS) e.g. data throughput, energy efficiency [1] [2]. The scheduler assigns network resources (i.e. channels) to User Equipments (UE's) based on certain set criteria. Most schedulers aim at maximizing the throughput, minimizing delays and improving resources allocation among users' fairness. This is challenging due to the rapid increase in the number of users (or devices) connecting the network [3].This increase is predicted to push the demand on existing mobile network to its limit. This is going to cause a reduction in network data rate performance and increase the energy consumption. This stimulates wireless research community to make considerable efforts on the enhancement the design of packet schedulers that can be used in the future networks. The future cellular networks are expected to provide ubiquitous broadband access to the growing populace of mobile users and also support a diversity of data services with high data rates [2].

Research in scheduling algorithms is growing as it appears to be a promising application for the next generation of wireless systems [2]. A smart scheduling scheme may provide enough flexibility to accommodate a number of channel and network conditions in deciding radio resource allocations. A packet scheduler can handle the effect of poor channel conditions by changing the amount of allocated resources per user and utilizing efficient modulation schemes [3]. The Generalized Proportional Fair $(G P F)$ scheduler was proposed in [4] and provides an effective solution for best effort traffic in a downlink. This strategy exploits multiuser diversity in the frequency and time domain by applying a fast-packet scheduling to Orthogonal Frequency Division Multiple Access (OFDMA) which is used for downlink.

In this paper, we study the GPF for LTE-A downlink and show the performance enhancements by comparing it with the State-of-the-Art (SOTA) LTE packet schedulers. The simulation results show a comparison between the $G P F$ and conventional schedulers in terms of the data throughput, energy efficiency, spectral efficiency and the fairness index. The GPF scheduler finds the right balance between fairness and throughput by tuning certain variables in the GPF metric. This tunning modifies the past achieved throughput and instantaneous data rates of users in order to increase the probability of allocation and serve users that satisfy current requirements and therefore achieve better network performance. An overview of the conventional scheduling algorithms is given in section II and section III explains the GPF scheduling algorithm. The system model is explained in section IV. Simulation results and related discussion is presented in section $\mathrm{V}$ and section VI concludes this paper.

\section{CONVENTIONAL SCHEdUlers FOR LTE-A}

In this section, we introduce three schedulers commonly used in the literature: Round Robin, Best Quality Indicator and Proportional Fair. The performance of SOTA schedulers is compared with the performance of GPF in section III.

\section{A. Round Robin (RR) Scheduler}

The $R R$ scheduler is a simple cyclical scheduler where resources are allocated to the users in turns. The $R R$ scheduler distributes network resources to the users equally irrespective of their channel conditions. Consequently, the system throughput is lower compared to other schedulers. However, it maintains a comparably good distribution of resources and fairness [2]. For the purpose of comparison, the RR scheduler is considered as a worst-case scenario in this paper. The scheduling metric for the $n$-th user on the $m$-th Resources Block (RB) can be given as [2]:

$$
\alpha_{n, m}^{R R}=\tau-T_{n}
$$


where $\tau$ is the current time slot, and $T_{n}$ is the most recent time slot when user $n$ is served.

\section{B. Best Quality Indicator (BCQI) Scheduler}

The $B C Q I$ scheduler aims to maximise the cumulative network throughput. Optimal network throughput performance is achieved by assigning the network channels to users that can achieve maximum throughput in the current time slot i.e. to the user that has the best channel quality. The cumulative cell throughput can be maximised by using BCQI scheduler, however, this comes at an expense of fairness. The users with poor channel conditions, such as the cell edge users, may get fewer resources, and hence a lower data throughput. The $B C Q I$ scheduler metric can be represented as:

$$
\alpha_{n, m}^{B C Q I}=C Q I_{m}^{n}(\tau)
$$

where $\alpha_{n, m}^{B C Q I}$ is the $B C Q I$ scheduler metric for the $n$-th user on the $m$-th $\mathrm{RB}, C Q I_{m}^{n}(\tau)$ is the reported Channel Quality Indicator (CQI) value of user $n$ in the current time $\tau$.

\section{Proportional Fair (PF) Scheduler}

The $P F$ scheduler aims at optimising the throughput and fairness in the network. The $P F$ scheduler distributes resources among users by taking into consideration their channel quality and their past average channel quality. In particular, it allocates resources to users that will maximize the ratio of the achievable instantaneous data rate over the average received data rate [2]. This uniquely satisfies the equity and fairness criteria [5] and thus, even the users with poor channel conditions would be served fairly. Therefore, the $P F$ scheduler delivers a good balance between fairness, spectral efficiency and throughput. The scheduler metric can be expressed as [2]:

$$
\alpha_{n, m}^{P F}=\frac{\rho_{n(\tau)}}{\rho_{n}(\tau-1)}
$$

where $\alpha_{n, m}^{P F}$ is the metric of the $n$-th user on the $m$-th resource block, $\rho_{n}(\tau-1)$ is the past average throughput achieved by the $i$-th user until time $\tau$ and $\rho_{n(\tau)}$ is the expected data rate for the $n$-th user at time $\tau$ on the $m$-th RB.

\section{GENERALIZED PROPORTIONAL FAIR (GPF) SCHEDULER}

In this section, the GPF scheduling algorithm is introduced. The $G P F$ scheduler resources allocation metric aims to balance between $B C Q I$ cell throughput performance and $P F$ throughput fairness performance [2]. The GPF metric and the enhancement process can be expressed as follows:

$$
\alpha_{n, m}^{P S}=\frac{\left[\rho_{n}(\tau)\right]^{\beta}}{\left[\rho_{n}(\tau-1)\right]^{\gamma}}
$$

where $\alpha_{n, m}^{P S}$ is the metric of the $n$-th user on the $m$-th RB, $\rho_{n}(\tau-1)$ is the past average throughput achieved by the $n$-th user until time $\tau, \rho_{n}(\tau)$ is the expected data rate for the $n$-th user at time $\tau$ on the $m$-th RB. The role of $\gamma$ and $\beta$ is to modify the impact of the past achieved throughput and instantaneous data rate respectively.

It is worth mentioning that, when $\gamma=0$, maximum throughput and maximum spectral efficiency is guaranteed at the expense of fairness. The metric modifies the weight of the past achieved throughput of UEs in the current time slot. If the past achieved throughput was higher, the scheduler would be able to allocate more resources to the UE to help them to achieve higher data rates. On the other hand, when $\beta=0$, an equal throughput scheduling scenario is achieved. The metric modifies the impact of the allocation policy on the expected instantaneous data rate for UEs in the network. With an equal rate for all users, the scheduler allocates resources to UEs in such a way that they achieve equal throughput [2].

The increasing value of $\beta$ increases the influence of the instantaneous data rate that can be achieved. Therefore, it improves the probability of serving a user with good channel conditions. This translates to higher network throughput but with a lower throughput fairness index. An increase in the metric $\gamma$ seeks to influence the past achieved throughput. This increases the chance of a user that has achieved lower throughput values to be scheduled, resulting in a low network throughput but a high fairness index can be predicted.

\section{SySTEM MODEL}

For the purpose of system level simulations, a standard compliant LTE simulator [6] is used in this paper. The simulator allows for the measurements of AMC feedback, MIMO gains and scheduling for individual UE physical layer links. This is because of the complex nature and computational power required to carry out radio link simulations between all base stations and terminals. A single simulation run operates in a given Region of Interest (ROI) and the eNB location is fixed and UEs are randomly distributed in the ROI throughout the simulation duration.

Table I shows the summary of key parameters used in the simulations. There is one main eNB with 3 sites to simulate three different cells. UEs were constantly placed for all the scenarios simulated using the fixed radial distribution method and 10 UEs were placed in each cell. The UEs were non-mobile in order to achieve fair comparison for all the considered schedulers. For simplicity, the simulation transmitter had one antenna and the receiver has one antenna ( $1 \times 1$ antenna). It is possible to get higher throughput values using Multiple Input Multiple Out (MIMO) technology but the scope of this paper is to compare the performance of each scheduler given the same conditions and therefore a $1 \times 1$ scenario is sufficient enough. After brute force testing all the values for $\beta$ and $\gamma$ we found that the optimal value for this simulation scenarios is 0.6 and 0.7 respectively.

Table I Simulation Parameters

\begin{tabular}{|l|l|}
\hline Parameter & Value \\
\hline Number of eNBs / cells & $1 / 3$ \\
\hline Number of UEs & 30 \\
\hline Channel Model & Typical Urban [7] \\
\hline Transmission Bandwidth & $20 \mathrm{MHz}$ \\
\hline
\end{tabular}




\begin{tabular}{|l|l|}
\hline Carrier Frequency & $2.1 \mathrm{GHz}$ \\
\hline User Distribution / Speed & Fixed Radial Distr. [8]/0 \\
\hline eNB Transmission Power & $46 \mathrm{dBm}$ \\
\hline Antenna Type & $1 \times 1$ SISO $[8]$ \\
\hline Simulation Type & Tri Sector \\
\hline Transmission Time Interval & $100 \mathrm{~ms}$ \\
\hline Schedulers & $R R, B C Q I, P F$ \\
\hline Network Geometry & Hexagonal Grid \\
\hline
\end{tabular}

The distribution of UEs location is shown in Fig. 1, showing the $\mathrm{x}$ and $\mathrm{y}$ position of the eNB and the UEs in the simulation environment. The distances of each UEs with the eNB has an impact on overall performance. To conduct a fair comparison, the same UE positions were used in all simulations. The eNB is located at the center of the ROI as shown in the Fig. 1 and UEs are distributed by a constant radial around the eNB.

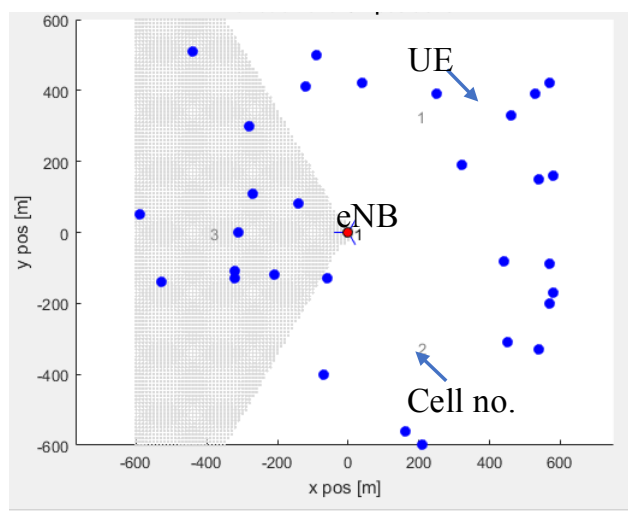

Fig. 1 The distribution of users and eNB in the ROI. The users are shown with the dots. The eNB is located in the middle of the ROI i.e. x,y position is 0,0 . The ROI contains three cells and the shaded area represents cell number three.

\section{GPF PERFORMANCE EVALUATION}

\section{A. Throughput Performance}

The average UE throughput performance of the GPF and the conventional schedulers in the simulation ROI is shown in Fig. 2. The Standard Error of Mean (SEM) bars for each scheduler is also shown in Fig. 2. The $G P F$ average UE throughput outperforms both the $R R$ and $P F$ schedulers. As expected, the GPF cannot outperform the average UE throughput of the $B C Q I$ scheduler. However, as shown by the standard error of the mean of the $B C Q I$, some UE are achieving a significantly lower throughput than the average performance. A further consequence of the BCQI throughput comparable high throughput performance is shown in the fairness index results.

\section{B. Energy per bit performance}

Fig. 3 represents the average energy used by UEs and the SEM under the different packet schedulers. Due to the allocation policy of the $B C Q I$ scheduler, it can be seen that it is highly energy efficient. To perform scheduling in BCQI, UEs compute their CQI values and send this information to the eNB. The CQI values computed by the UE contains information regarding the Signal to Noise and Interference ratio. A high CQI value will indicate that the UE is experiencing good channel conditions. Therefore, UEs scheduled under the BCQI method will have a lesser need for power and energy consumption per bit of information transmitted because of the low noise and interference on the channel propagation path. The $B C Q I$ scheduler shows a better energy efficiency when compared to the rest of the schedulers and, in particular, the GPF, but it should be noted that this is because the GPF does not ignore users experiencing bad channel condition. The energy efficiency levels achieved by the GPF scheduler is good enough to support UEs in LTE-A networks.

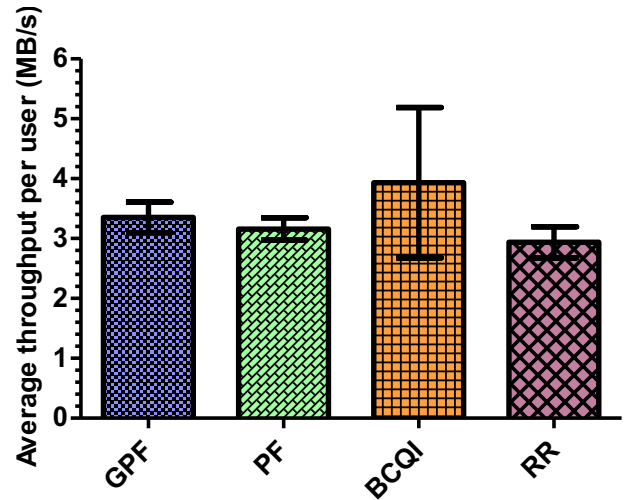

Fig. 2 Average users' throughput performance showing the GPF, PF, BCQI and RR schedulers. The error bars represent the standard error of the mean.

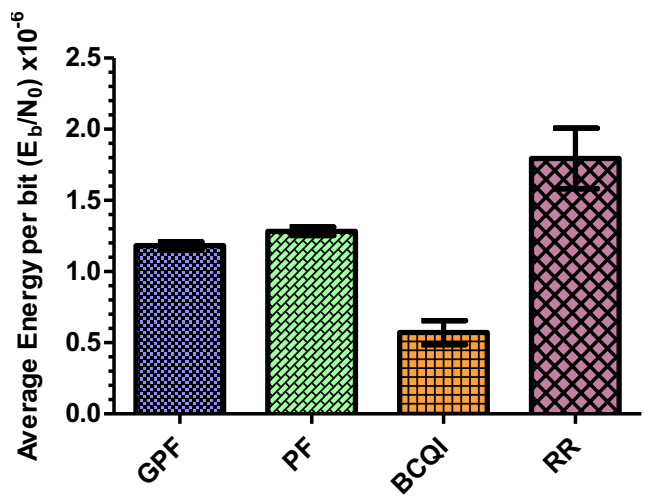

Fig. 3 Average Energy per bit performance of the $G P F, P F, B C Q I$ and $R R$ schedulers. The standard error of the mean is also shown.

\section{Spectral Efficiency performance}

The spectral efficiency is essential in order to properly utilize the scarce and expensive radio resources. In this paper, the spectral efficiency is expressed in bits per channel use (bits/cu) i.e. the number of bits that can be transmitted over a channel for a particular duration of time. It is efficient when more users are served in a given time and all can achieve maximum performance with the resources allocated to them. Schemes that make use of aggressive resource reuse strategies to ensure efficient use of expensive radio resources classified as spectral efficient schedulers.

From the simulation results of four schedulers in Fig. 4, it can be seen that the performance of $G P F$ scheduler is almost same as the performance of the $B C Q I$. This difference, although not negligible, is tolerable. The results are so because the $B C Q I$ scheduler only assigns radio resources to users that are experiencing good channel condition. 


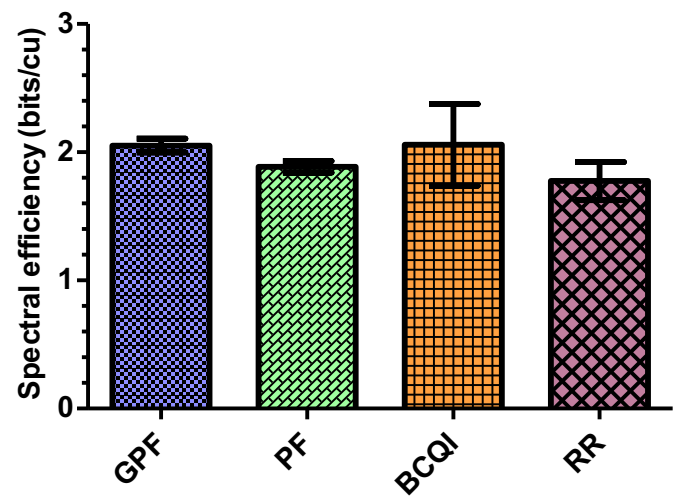

Fig. 4 Spectral efficiency of the $G P F, P F$, BCQI and $R R$ schedulers. The standard error of mean is shown.

\section{Fairness Index performance}

The Jains fairness index is used to evaluate the achieved throughput fairness as shown in Fig.5. The $P F$ scheduler shows the highest fairness (Jain's fairness Index of 0.9). The GPF scheduler achieves slightly lower fairness performance i.e. Jain's fairness index of 0.86 . The $B C Q I$ scheduler shows the worst fairness (Jain's fairness index of 0.25 ) because it completely ignores UEs experiencing bad channel conditions.

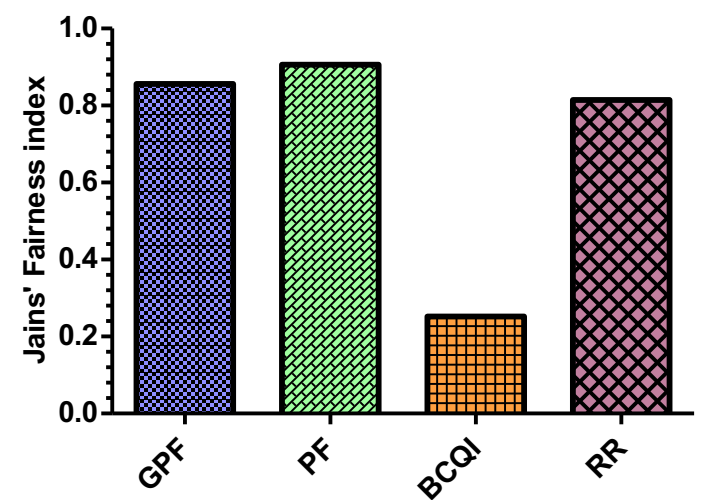

Fig. 5 Jains fairness index performance of the $G P F, P F, B C Q I$ and $R R$.

Although the GPF performs slightly lower fairness performance and average energy per bit performance, it outperforms the PF in the average UE throughput and spectral efficiency. Furthermore, the sum of the achieved throughput and average energy per bit of the GPF outperforms the PF as shown in Fig. 6. Table II summarizes the simulation results by showing the order of the scheduler performance with respect to the other schedulers. In other words, in Table 2 the scheduler which has one for a specific performance metric means that it outperforms the other schedulers.

Table II The simulation results summary, showing the order of the scheduler with respect of the other schedulers.

\begin{tabular}{|l|c|c|c|c|}
\hline Performance metric $\backslash$ Scheduler & GPF & PF & BCQI & RR \\
\hline Average throughput per UE & 2 & 3 & 1 & 4 \\
\hline Average energy per bit & 2 & 3 & 1 & 4 \\
\hline Spectral efficiency & 2 & 3 & 1 & 4 \\
\hline Jains Fairness index & 2 & 1 & 4 & 3 \\
\hline Sum UEs throughput & 2 & 3 & 1 & 4 \\
\hline Sum of UEs energy per bit & 2 & 3 & 1 & 4 \\
\hline
\end{tabular}

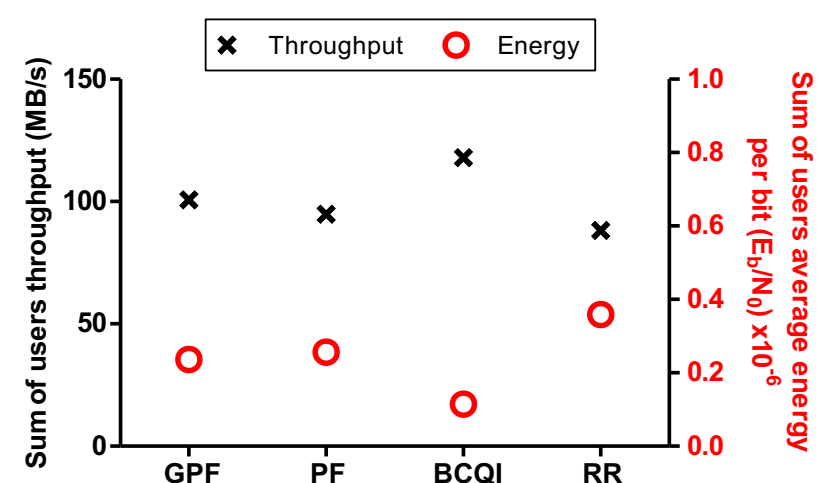

Fig. 6 The sum of the users' throughput and average energy per bit for the $G P F, P F$, BCQI and $R R$ schedulers.

\section{CONCLUSION}

In this paper, we presented the performance of Generalized Proportional Fair scheduler using a system level standard compliant simulator. We further compared the GPF scheduler to the other conventional schedulers for a LTE-A network. The simulation results show that the $G P F$ scheduler outperforms the Proportional Fair scheduler in the average throughput per user, average energy per bit, spectral efficiency and sum of the users' throughput and energy per bit. On the other hand, the Proportional Fair outperforms the Generalized Proportional Fair, Best Channel Quality Indicator and the Round Robin schedulers in the Jains fairness index performance.

\section{REFERENCES}

[1] M. Dehghani, K. Arshad, and R. MacKenzie, "LTE-Advanced Radio Access Enhancements: A Survey," Wirel. Pers. Commun., vol. 80, no. 3, pp. 891-921, 2014.

[2] F. Capozzi, G. Piro, L. A. Grieco, G. Boggia, and P. Camarda, "Downlink packet scheduling in LTE cellular networks: Key design issues and a survey," IEEE Commun. Surv. Tutorials, vol. 15, no. 2, pp. 678-700, 2013.

[3] B. Barakat and K. Arshad, "An adaptive hybrid scheduling algorithm for LTE-Advanced," in 2015 22nd International Conference on Telecommunications (ICT), 2015, pp. 91-95.

[4] C. Wengerter, J. Ohlhorst, and A. G. E. Von Elbwart, "Fairness and throughput analysis for generalized proportional fair frequency scheduling in OFDMA," 2005 IEEE 61st Veh. Technol. Conf., vol. 3, no. 2, pp. 0-4, 2005.

[5] A. Mishra and P. Venkitasubramaniam, "Anonymity and fairness in packet scheduling: A quantitative tradeoff," IEEE/ACM Trans. Netw., vol. 24, no. 2, pp. 688-702, 2016.

[6] C. Mehlführer, J. Colom Ikuno, M. Šimko, S. Schwarz, M. Wrulich, and M. Rupp, "The Vienna LTE simulators - Enabling reproducibility in wireless communications research," EURASIP J. Adv. Signal Process., vol. 2011, no. 1, p. 29, 2011.

[7] H. Asplund, K. Larsson, and P. Ökvist, "How typical is the 'typical urban' channel model? - Mobile-based delay spread and orthogonality measurements," in IEEE Vehicular Technology Conference, 2008, pp. 340-343.

[8] Vienna University of Technology (Institute of Telecommunications), "Vienna LTE Simulators System Level Simulator Documentation," v1.6r885, Web: http://www.nt.tuwien.ac.at/ltesimulator, pp. 1-14, 2010. 